\title{
Performance-Optimal Clustering with Retiming for Sequential Circuits *
}

\author{
Tzu-Chieh Tien and Youn-Long Lin \\ Department of Computer Science, Tsing Hua University \\ Hsin-Chu, Taiwan 30043, R.O.C. \\ Tel, Fax: 886-3-573-1072 \\ E-mail: $\{$ dr814323,ylin\}@cs.nthu.edu.tw
}

\begin{abstract}
We propose an exact clustering with retiming algorith$\mathrm{m}$ to minimize the clock period for sequential circuits. Without moving flip-flops (FF's) by retiming, conventional clustering algorithms can only handle combinational parts and therefore cannot achieve the best cycle time. Pan et al. [2] have proposed an optimal algorithm under the unit gate delay model. We propose a more powerful and faster algorithm that produces optimal results even under the more realistic general gate delay model. Experimental results show that our algorithm is twice as fast as Pan's.
\end{abstract}

\section{Introduction}

Circuit clustering groups cells in a design into macros to satisfy some constraints such as area or pin limitations $[3][4][5]$. But this often induces large interconnect delays between macros. Therefore, avoiding performance degradation is a major objective when we perform circuit clustering.

Retiming, which repositions flip-flops (FF's) while preserving circuit functionality, can be used to shorten the clock period [1]. Traditional clustering techniques, which do not consider retiming, cannot achieve the optimal performance for sequential circuits [4][5]. These clustering methods often treat a sequential circuit as combinational parts by dropping all FF's and then clustering each combinational part independently. If we can appropriately relocate FF's by retiming when clustering a circuit, we can achieve better performance.

Pan et al. have proposed an approach to combine retiming and clustering[2]. Under the unit gate delay model[4], their algorithm can achieve the optimal clock period. For the general gate delay model, it can get near-optimal clock period within the maximum delay of any gates in the circuit. They use a labeling technique to achieve the retiming effect and to integrate it with clustering.

Pan's algorithm cannot produce the optimal results under the general delay model because it does not find the best labeling. If the relocated position for an FF, computed during labeling, is occupied by a gate, the labeling value of this gate has to be modified.

${ }^{*}$ This work was supported in part by the Science Council R.O.C., under a contract no. NSC88-2215-E-007-012.
In this paper, we propose a new algorithm to cluster circuits with retiming using a new labeling method. This algorithm not only can achieve the optimal clock period under the general delay model but also uses less time than Pan's. Experimental results show that the average ratio of the run time used by Pan's algorithm to ours is $2: 1$.

The rest of this paper is organized as follows. Preliminaries are described in Section 2. How to enhance Pan's labeling method is presented in Section 3. For the convenience of illustrating our clustering algorithm, we first review Pan's algorithm in Section 4. Our algorith$\mathrm{m}$ is introduced in Section 5. Section 6 presents some experimental results. Finally, Section 7 draws some concluding remarks.

\section{Preliminaries}

A sequential circuit $N$ is modeled as a directed (multi)graph $G(V, E, d, w)$. Each vertex $v \in V$ corresponds to a combinational gate, a primary input (PI), or a primary output $(\mathrm{PO})$ with propagation delay $d(v)$. Each directed edge $e \in E$ connects two vertices $u$ and $v$ if the corresponding gate of $u$ drives, across zero or more registers, an input of the corresponding gate of $v$. The number of registers between $u$ and $v$ is denoted as edge weight $w(e)$ or $w(u, v)$. A path $p$ consists of a sequence of successive vertices and edges such as $p=v_{0} \stackrel{e_{0}}{\rightarrow} v_{1} \stackrel{e_{1}}{\rightarrow} \cdots \stackrel{e_{k-1}}{\rightarrow} v_{k}$ and is denoted as $v_{0} \stackrel{p}{\sim} v_{k}$. The path delay is the sum of the delay of each vertex of $p: d(p)=\sum_{i=0}^{k} d\left(v_{i}\right)$. The path weight is the sum of the edge weight of $p$ : $w(p)=\sum_{i=0}^{k-1} w\left(e_{i}\right)$. The critical path of the circuit has the maximum zero-weight path delay denoted as $\phi(G)=\max \{d(p): w(p)=0\}$.

A retiming of a circuit is an integer-valued vertexlabeling function $r: V \rightarrow Z$. Labeling vertex $v$ with $r(v)$ means that $r(v)$ registers will be moved from each output and added to each input of $v$ as shown in Fig. 1. The edge weight of the retimed graph $G_{r}\left(V, E, d, w_{r}\right)$ can be obtained by

$$
w_{r}(e)=w(e)+r(v)-r(u)
$$

A clustered circuit is composed of clusters and is functionally equivalent to the original one. The area of each cluster must be less than $M$, the given area constraint. The interconnect between clusters incurs large 


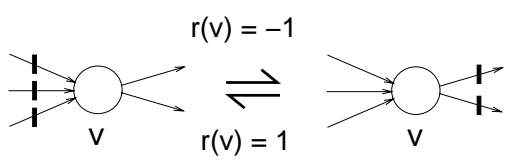

Fig. 1: Basic retiming operation.

delay, $D$, which is also a given parameter. We define the function $\lambda(u)$ to denote the interconnect delay from $u$ to a cluster. Because we assume there is no inter-cluster delay for PI's and PO's, $\lambda(u)$ is zero if $u$ is a PI or the cluster is a PO; otherwise it is $D$. A node can be duplicated without changing functionality for optimizing the clock period. The cycle time may differ from the original because of retiming. The clustering problem in this paper is as follows:

Problem 1 Given a sequential circuit $G$, and a target clock period c, find a clustered circuit $G_{r}$ with $\phi\left(G_{r}\right)$ less than or equal to $c$, if such a circuiting exists.

\section{The Weakness of Pan's Approach}

In this section, we explain why Pan's approach cannot obtain the optimal clock period under the general delay model and give our solution. Pan's approach does not find the best labeling. The algorithm labels each node in the circuit an "l-value", defined as the weight of the longest path from the PI's to the node using the " $w_{1}$ weight". The $w_{1}$ weight is defined as $w_{1}(e)=-c *$ $w(e)+d(v)$ for each edge $e: u \rightarrow v$, where $c$ is the target clock period.

Next we explain the meaning of the l-value. Consider a path $p$ starting from a PI $u$ and ending at a node $v$. If we want to retime the path to satisfy the time constraint $c$, there must be at least $\lceil d(p) / c\rceil-1 \mathrm{FF}$ 's on $p$ in the final circuit. Since there exists $w(p)$ FF's on $p$, we can set the retiming value $r(v)$ as $(\lceil d(p) / c\rceil-1)-w(p)$. $(\lceil d(p) / c\rceil-1)-w(p)$ can be further translated as follows:

$$
\begin{aligned}
& (\lceil d(p) / c\rceil-1)-w(p) \\
= & \lceil d(p) / c-w(p)\rceil-1 \\
= & \lceil(d(p)-c * w(p)) / c\rceil-1 .
\end{aligned}
$$

We want to use a function related to $v$ to represent the value $d(p)-c * w(p)$, so we let

$$
l(v)=d(p)-c * w(p) .
$$

If $p$ is the longest path from the PI's to $v$ using the $w_{1}$ weight, $l(v)$ is the l-value. The use of l-value also gives a good failure condition for the retiming algorithm by checking on whether the l-value of the $\mathrm{PO}$ is greater than $c$ [2]. If the l-value of a PO is greater than $c$, there exists no clustered circuit $G_{r}$ with $\phi\left(G_{r}\right)$ less than or equal to $c$.

However, this labeling method is not adequate if we want to find the optimal solution under the general delay model. In fact, it attempts to relocate the $i$ th FF to the position at which the propagation delay from PI equals to $i * c$. Unfortunately, this position is very likely to be at the middle of a gate. Since a gate cannot be split, we can only push this FF to the front of the gate to satisfy the timing constraint $c$. Thus this kind of approach may produce a solution near the optimal within the maximum delay of any gates in the circuit.

In our approach, we follow the method proposed in [6] to modify the l-value. The l-value of a node $v$ can be computed by using the l-value of its fan-in node $u$ by

$$
l(v)=l(u)-c * w(u, v)+d(v) .
$$

If we find that there is an $\mathrm{FF}$ with computed position occupied by a gate $v$, the l-value of $v$ is increased to

$$
\operatorname{lag}(l(v)) * c+d(v),
$$

where $\operatorname{lag}(x)$ is defined as

$$
\operatorname{lag}(x)=\lceil x / c\rceil-1 .
$$

We can detect whether the computed position of an FF is occupied by a gate $v$ by checking on whether $\operatorname{lag}(l(v))$ is greater than $\operatorname{lag}(l(v)-d(v))$. The optimal-clockperiod circuit can be obtained by setting the retiming value $r(v)$ for each $v$ according to the final l-value as

$$
r(v)= \begin{cases}0 & \text { if } v \text { is a PI } \\ \operatorname{lag}(l(v)) & \text { otherwise. }\end{cases}
$$

Fig. 2 shows a simple example that Pan's approach cannot achieve the optimal clock period but we can. Fig. 2 (a) is the original circuit showing the gate delay on each node. The minimal timing constraint that Pan's labeling can achieve is 5. The l-value and the corresponding retiming value $r(v)$ for each node are listed in Fig. 2 (b). However, the critical path delay of the

\begin{tabular}{|c|c|c|c|c|c|c|c|c|}
\hline $\begin{array}{l}\text { l-value } \\
\text { with } c=5\end{array}$ & $\mathbf{0}$ & 2 & -1 & 1 & 3 & 6 & 8 & 5 \\
\hline$r(v)$ & $\mathbf{0}$ & 0 & -1 & 0 & 0 & 1 & 1 & 0 \\
\hline
\end{tabular}
retimed circuit is 7 . On the other hand, the minimal timing constraint that our labeling can achieve is 6 . As

\begin{tabular}{|c|c|c|c|c|c|c|c|c|}
\hline $\begin{array}{l}l-\text { value } \\
\text { with } c=6\end{array}$ & $\mathbf{0}$ & 2 & -2 & 0 & 2 & 5 & 8 & 4 \\
\hline$r(v)$ & $\mathbf{0}$ & 0 & -1 & -1 & 0 & 0 & 1 & 0 \\
\hline
\end{tabular}
shown in Fig. 2 (c), the critical path delay of the retimed circuit according to our labeling is 6 , which is the optimal clock period.

$$
\text { original } \longrightarrow \text { (2) } \mathbf{H} \rightarrow(2) \rightarrow(2) \rightarrow(3) \rightarrow(2) \rightarrow
$$

Fig. 2: (a) The original circuit. (b) The final critical path delay is 7 by using Pan's labeling. (c) We can achieve the optimal clock period 6 by using our labeling. 


\section{Outline of Pan's Algorithm}

For illustrating our algorithm, we outline the algorithm proposed by Pan et al.[2] in this section. Their algorith$\mathrm{m}$ consists of two phases. The first is the labeling phase to compute the l-value and generate the corresponding cluster for each node. The second phase connects al1 nodes and clusters, retimes the clustered graph, and then merges clusters to reduce area. Clusters have to be merged because the algorithm generates one cluster for each node during the first phase. We address the labeling phase in this paper; the second phase can be found in [2].

As we have illustrated in the previous section, the l-value of a PO cannot be greater than the target clock period $c$. Instead of Problem 1, Pan's algorithm tries to solve the "Strong Clustering Problem":

Problem 2 (Strong Clustering Problem) Given a positive integer $c$, find a clustered circuit such that the $l$ values of the PO's in the circuit are less than or equal to $c$.

To reduce the search space, Pan's algorithm only considers "simple clustered circuits". A simple clustered circuit satisfies four conditions:

1. Each cluster has only one node that can output signals to the outside of the cluster. Thus we can name a cluster as $C_{v}$ if it outputs from node $v$.

2. The retiming value at each node in the clustered circuits is zero.

3. For each node $v$ in the circuit, there is at most one $C_{v}$.

4. If $C_{u}$ is connected to nodes in $C_{\vartheta}$, then $C_{\vartheta}$ does not contain a copy of $u$.

Details about the simple clustered circuit can be found in [2]. We just quote one of its theorems:

Theorem 1 If a strong clustering problem has a solution, it has a simple solution.

Pan's algorithm iteratively computes a new l-value for each node. A new cluster $C_{v}$ which leads to the new l-value for node $v$ is generated at the same time. If the present l-value of $v$ is less than the new computed value, it is updated. If no more l-value can be updated, the algorithm terminates. Otherwise, the failure condition will be met for some $\mathrm{PO}$ with its l-value greater than the target clock period $c$. Fig. 3 gives the pseudo code of Pan's labeling procedure.

The new l-value of a node $v$ and the new cluster $C_{v}$ are calculated by the routine PANSTIGHTENBOUND as shown in Fig. 4. PANsTightenBound first computes the candidate l-value of $v$ for each $u$ by assuming that $u$ fanouts to $C_{v} . l^{\prime}(u)$, the candidate l-value of $v$ for each $u$, is computed by the equation:

$$
l^{\prime}(u)=l(u)+\Delta(u, v)+\lambda(u)
$$

where $\Delta(u, v)$ is the $w_{1}$ weight of the longest path from $u$ to $v$. Among these candidates, there exists an $l^{\prime}(u)$ which may become the new l-value of $v$. So, a binary search is performed to find the minimum $l^{\prime}(u)$ and

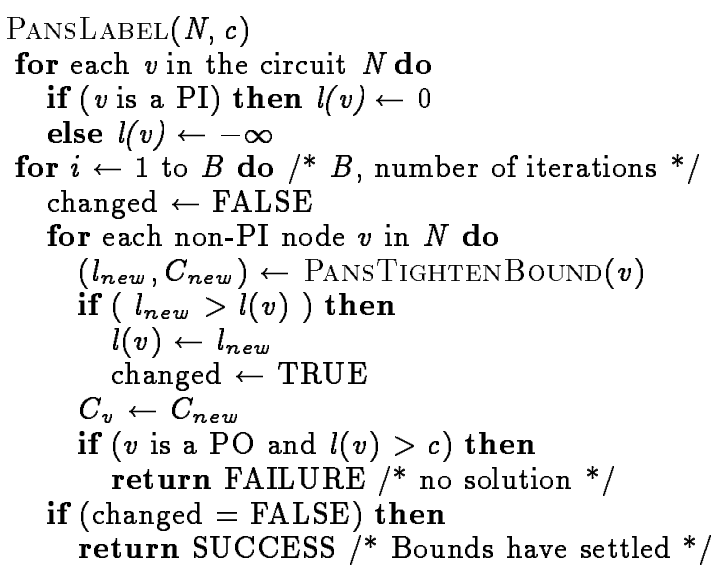

Fig. 3: Pan's labeling procedure.

the legal cluster $C_{v}$ leading to the $l^{\prime}(u)$. If we want to achieve a desired l-value $L$ for $v$, there is no choice but putting all nodes with $l^{\prime}(u)^{\prime} s$ greater than $L$ into the cluster $C_{v}$. Of course, the total area of the formed cluster $C_{v}$ must be smaller than $M$, the given cluster size constraint. Finally, PANSTightenBound will return a possible l-value for $v$ to be compared with the present one and a legal cluster $C_{v}$.

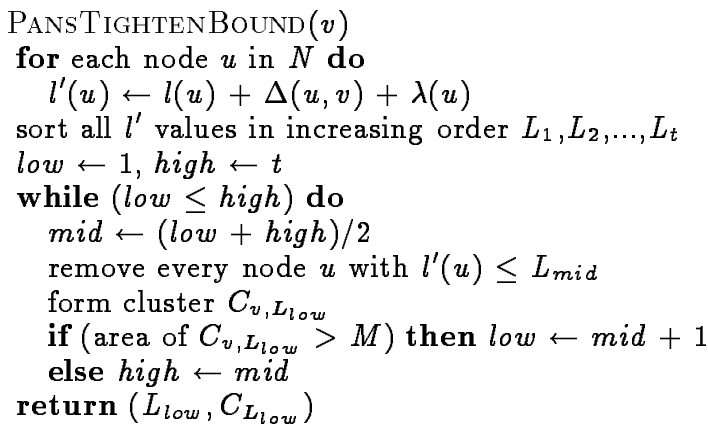

Fig. 4: Pan's procedure for improving the lower bound.

The time used by Pan's algorithm is mainly spent on $\Delta(u, v)$ calculation and the labeling phase. $\Delta(u, v)$ can be calculated in time $O\left(|V|^{2} \log |V|+|V||E|\right)$ by using an all-pair shortest path algorithm [10]. Time for the labeling phase depends on how many iterations will be executed (i.e., $B$ in Fig. 3). Pan et al. give the upper bound on $B$ as $|V|(|V|-1) D$, and PansTightenBound takes time $O((|V|+|E|) \log |V|)$. Therefore, the time complexity for the algorithm is $O\left(|V|^{3}|E| D \log |V|\right)$. 


\section{Proposed Algorithm}

In this section, we present our exact algorithm for the performance-optimal clustering with retiming. We have illustrated our labeling method leading to the optimal clock period in Section 3 with

$$
\begin{gathered}
\text { new } 1-\text { value of } v= \\
\left\{\begin{array}{l}
\operatorname{lag}(l(v)) * c+d(v) \quad \text { if } \operatorname{lag}(l(v))>\operatorname{lag}(l(v)-d(v)) \\
l(u)-c * w(u, v)+d(v) \text { otherwise. }
\end{array}\right.
\end{gathered}
$$

In addition, we adopt another circuit traversing method rather than Pan's by using FIFO's to incorporate our labeling technique for run-time efficiency.

The procedure PANSLABEL is a variation of the Bellman-Ford algorithm ${ }^{1}$ [10]. We develop an algorith$\mathrm{m}$ similar to the retiming algorithm proposed by Chen [6]. Chen's algorithm uses a FIFO to store the nodes for updating rather than iteratively traversing the whole circuit. Experimental results show that Chen's algorith$\mathrm{m}$ runs much faster than the Bellman-Ford-like retiming algorithm [6]. Thus in our labeling procedure, we use a queue, called queue1, to store the nodes whose new l-values will be calculated. Initially, all PI's are put into queue1. Then, nodes are retrieved from queue 1 one at a time. We update the l-value for a node if the calculated value is greater than the present one. Then we put all nodes reachable from the updated node into queue 1 if they have a chance to be updated. The procedure stops if queue 1 is empty or the failure condition is detected. Fig. 5 shows the procedure, LABEL.

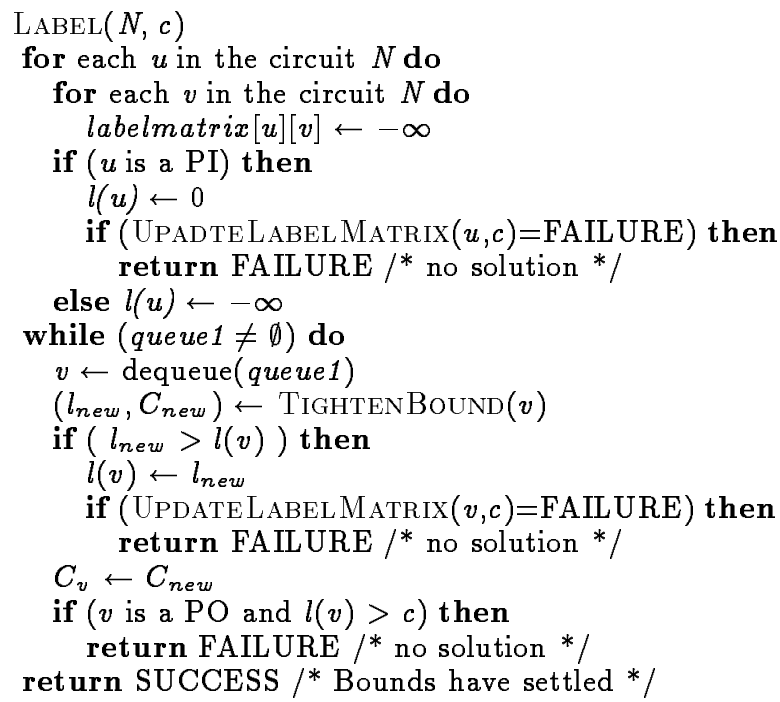

Fig. 5: Our labeling procedure.

Fig. 6 shows routine UPDATELABEL MATRIX which decides on whether a node has a chance to be updated. Since a node reachable from the updated vertex

${ }^{1}$ Strictly speaking, PANSLABEL is not a pure Bellman-Ford algorithm because a failure condition is inspected to enhance the run-time efficiency.

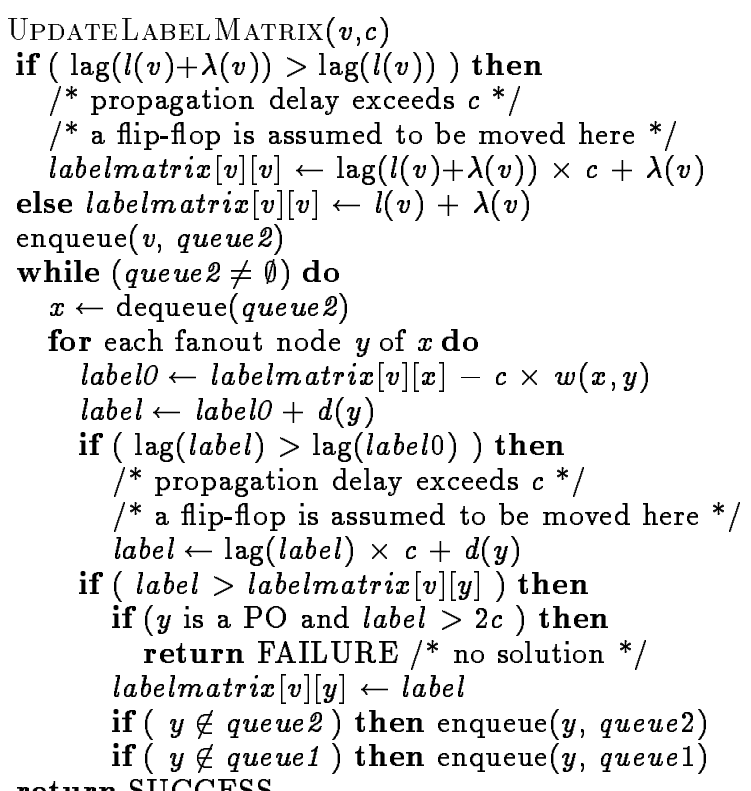

return SUCCESS

Fig. 6: Our procedure for updating the labelmatrix.

$v$ may have a chance to be updated, we use another queue, named queue2, to help traverse the sub-circuit starting from $v$. If the l-value of a node $y$ computed according to the updated l-value of $v$ is greater than the original value, $y$ will be put into queue 2 for further traversal. The candidate l-value (i.e., $l^{\prime}(v)$ ) to a node $y$ computed according to the l-value of node $v$ is stored in labelmatrix $[v][y]$. The l-value is calculated according to Equation 1. Meanwhile, $y$ is also put into queue 1 if it is put into queue 2 since its l-value has a chance to be updated. Routine TightENBOUND as shown in Fig. 7 will calculate the possible l-value of $y$ associated with $C_{y}$ when $y$ is retrieved from queue1.

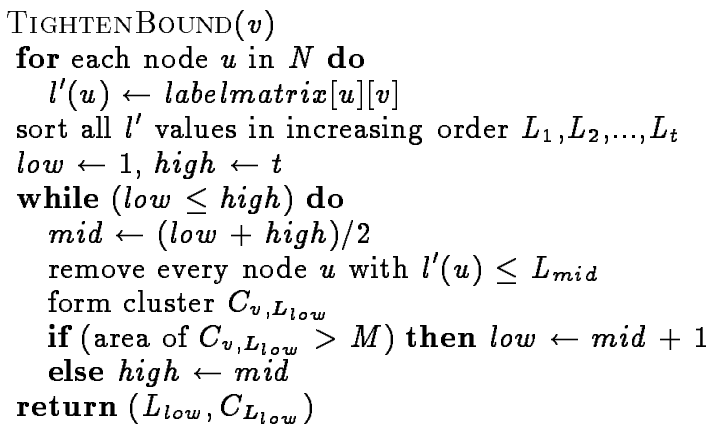

Fig. 7: Our procedure for improving the lower bound.

To prove the correctness of our algorithm, we give 
the following theorem:

Theorem 2 Given a sequential circuit $G$ and a target clock period $c$, there exists a retimed clustered circuit $G_{r}$ with $\phi\left(G_{r}\right)$ less than or equal to $c$ if, and only if, procedure LABEL returns SUCCESS.

The proof for Theorem 2 is omitted due to space limitation.

The storage requirement is $O\left(|V|^{2}\right)$ for the all-pairs matrix, labelmatrix, which is the same as that of Pan's algorithm for storing $\Delta(u, v)$ for every node pair $(u, v)$.

To analyze the time complexity, we first examine how many times a node will be visited in procedure $\mathrm{LA}^{-}$ BEL. A node $v$ will be visited only when the l-value of another node $u$ is updated and there is a path from $u$ to $v$. The increasing amount for an updated l-value is at least $c / \delta$, where $\delta$ is the minimal positive difference between any two gate delays. The l-value has a range between $-c *|F|$ and $c *(|F|+1)$ because the largest possible propagation delay is $c *(|F|+1)$, where $|F|$ is the number of FF's in the circuit. As a consequence, queue 1 is enqueued at most $\frac{c *(2|F|+1)}{\delta}|V|^{2}=$ $O\left(\delta^{-1}|F||V|^{2}\right)$ times. Next we examine how many times a node will be visited in routine UPDATELABEL MATRIX. A node $y$ will be visited only when one of its fan-in node, $x$, has been updated. By the similar analysis, $O\left(\delta^{-1}|F||E|\right)$ nodes will be visited in UPDATELABELMATRIX. Since the time complexity of TIGHTENBOUND is almost the same as PANSTIGHTENBound at $O((|V|+$ $|E|) \log |V|)$, our approach takes time $O\left(\left(\delta^{-1}|F||V|^{2}\right)\right.$ * $\left.\left.\left(\delta^{-1}|F||E|\right)+(|V|+|E|) \log |V|\right)\right)=O\left(\delta^{-2}|F|^{2}|V|^{2}|E|+\right.$ $\left.\delta^{-1}|F||V|^{2}|E| \log |V|\right)$. Comparing our results with the time complexity of Pan's approach, we are not sure which is faster. Thus we need experiments to prove the efficiency of our algorithm.

\section{Experimental Results}

We have implemented our algorithm in C language and embedded it in the SIS package [7]. We have also implemented Pan's algorithm for comparison purpose. We run the experiments on an UltraSPARC- 2 machine with $2 \mathrm{~GB}$ of memory. The $M$ is set as a quarter of the total circuit area, and $D$ twice the average gate delay.

Experimental results on the ISCAS89 benchmark suite [9] are listed in Table 1 . All the circuits are technology mapped by SIS using a $0.5 u m$ library from TSM$\mathrm{C}$ [8]. The number of gates for each circuit are listed in " $|V|$ ". Circuits are retimed before clustering and the results are listed in " $\phi(G)$ " column. The maximal gate delay of each circuit is listed in column "max $d$ " for reference. The minimal target clock periods that the labeling phase can achieve are listed in " $\min c$ " columns. The final clock periods are listed in " $\phi\left(G_{r}\right)$ " columns. Results prove that the clock period obtained by Pan's algorithm is bounded by $c$ plus maximal gate delay and may not be the optimal. 11 of totally 26 cases of Pan's results are sub-optimal as bold-faced in the table.

The run time in second is listed in "CPU" columns, and the ratio of the time used by Pan's algorithm over ours is listed in "Pan's/Ours CPU" column. Only for two cases (s1423 and s9234) is our algorithm a little bit slower than Pan's. The average ratio of the run time used by Pan's algorithm to ours is $2: 1$.
The experimental results prove our algorithm valuable since it is an exact algorithm and runs faster than the previous non-optimal heuristic.

\section{Conclusions}

We have presented an exact algorithm to cluster-withretiming sequential circuits to get the optimal clock period. We pointed out why a previous work, Pan's approach, could not produce the optimal solution under the general delay model. We modified Pan's labeling methods and proposed an exact algorithm which used two queues to enhance run-time efficiency. Experimental results show that the average ratio of the run time used by Pan's algorithm to ours is $2: 1$.

These techniques can also be used for technology mapping with retiming. We will study this problem in the future.

\section{References}

[1] C. E. Leiserson, and J. B. Saxe, "Retiming Synchronous Circuitry," Algorithmica, vol.6, pp.5-35, June, 1991.

[2] P. Pan, A. K. Karandikar, and C. L. Liu, "Optimal Clock Period Clustering for Sequential Circuits with Retiming," IEEE Transactions on ComputerAided Design of Integrated Circuits and Systems, vol.17, pp.489-498, June 1998.

[3] H. H. Yang, and D. F. Wong, "Circuit Clustering for Delay Minimization under Area and Pin Constraints," IEEE Transactions on Computer-Aided Design of Integrated Circuits and Systems, vol.16, pp.976-986, September 1997.

[4] R. Murgai, R. Brayton, and A. SangiovanniVincentelli, "On Clustering for minimum delay/area," in Proceedings of International Conference on Computer Design, pp.6-9, 1991.

[5] E. Lawler, K. Levitt, and J. Turner, "Module Clustering to Minimize Delay in Digital Networks," IEEE Transactions on Computers, vol.18, pp.4757, January 1969.

[6] W.-J. Chen, "A Study on the Relationship Between Retiming and Loop Folding," Master thesis, M312.93, National Tsing-Hua University, Taiwan, August 1994.

[7] E. M. Sentovich, K. J. Singh, L. Lavagno, C. Moon, R. Murgai, A. Saldanha, H. Savoj, P. R. Stephan, R. K. Brayton, and A. Sangiovanni-Vincentelli, "SIS : A System for Sequential Circuit Synthesis," Memorandum No. UCB/ERL M92/41, Electronics Research Laboratory, College of Engineering, University of California, Berkeley, May 1992.

[8] TCB650 Library, 0.5um Standard Cell Data Book, TSMC, April 1996.

[9] F. Brglez, D. Bryan, and K. Kozminski, "Combinational profile of sequential benchmark circuits," in Proceedings of International Symposium of Circuits and Systems, pp. 1929-1934, May 1989.

[10] T. H. Cormen, C. E. Leiserson, R. L. Rivest, Introduction to Algorithms, New York: McGraw-Hill, 1993. 
Table 1: Experimental results.

\begin{tabular}{|c|c|c|c|c|c|c|c|c|c|c|}
\hline \multirow{2}{*}{ circuit } & \multicolumn{3}{|c|}{ Initial } & \multicolumn{3}{|c|}{ Pan's } & \multicolumn{3}{|c|}{ Ours } & \multirow{2}{*}{$\begin{array}{l}\frac{P \text { Pan's }^{\prime}}{\text { Ours }} \\
\text { CPU }\end{array}$} \\
\hline & $|V|$ & $\phi(G)$ & $\max d$ & $\min c$ & $\phi\left(G_{r}\right)$ & $\mathrm{CPU}$ & $\min c$ & $\phi\left(G_{r}\right)$ & $\overline{\mathrm{CPU}}$ & \\
\hline s208 & 83 & 2.74 & 0.42 & 3.08 & 3.08 & 2.0 & 3.08 & 3.08 & $\overline{1.0}$ & 2.0 \\
\hline s298 & 98 & 0.99 & 0.38 & 0.99 & 1.05 & 3.9 & 1.05 & 1.05 & 2.4 & 1.6 \\
\hline s344 & 180 & 2.10 & 0.42 & 2.10 & 2.13 & 11.9 & 2.10 & 2.10 & $\overline{8.0}$ & 1.5 \\
\hline s349 & 174 & 1.99 & 0.42 & 1.99 & 1.99 & 11.1 & 1.99 & 1.99 & 6.9 & 1.6 \\
\hline s382 & 204 & 1.40 & 0.38 & 1.40 & 1.52 & 21.9 & 1.45 & 1.45 & 21.2 & 1.0 \\
\hline s386 & 175 & 2.30 & 0.38 & 2.66 & 2.66 & 7.3 & 2.66 & 2.66 & 4.2 & 1.7 \\
\hline $\mathrm{s} 400$ & 198 & 1.69 & 0.42 & 1.69 & 1.77 & 24.3 & 1.69 & 1.69 & 9.7 & 2.5 \\
\hline $\mathrm{s} 420$ & 179 & 2.80 & 0.42 & 3.15 & 3.15 & 6.2 & 3.15 & 3.15 & 2.7 & 2.3 \\
\hline $\mathrm{s} 444$ & 197 & 1.54 & 0.38 & 1.54 & 1.65 & 19.0 & 1.56 & 1.56 & 10.6 & 1.8 \\
\hline $\mathrm{s} 510$ & 288 & 2.41 & 0.70 & 2.58 & 2.90 & 40.4 & 2.79 & 2.79 & 22.6 & 1.8 \\
\hline s526 & 198 & 1.45 & 0.38 & 1.45 & 1.50 & 11.7 & 1.45 & 1.45 & $\overline{8.2}$ & 1.4 \\
\hline s526n & 185 & 1.46 & 0.42 & 1.46 & 1.49 & 13.9 & 1.46 & 1.46 & 6.7 & 2.1 \\
\hline s641 & 276 & 2.99 & 0.56 & 3.55 & 3.55 & 20.1 & 3.55 & 3.55 & 9.9 & 2.0 \\
\hline s713 & 225 & 2.35 & 0.38 & 2.69 & 2.69 & 10.3 & 2.69 & 2.69 & 4.7 & 2.2 \\
\hline s820 & 425 & 2.33 & 0.38 & 2.43 & 2.59 & 180.2 & 2.49 & 2.49 & 81.7 & 2.2 \\
\hline s832 & 428 & 2.33 & 0.38 & 2.50 & 2.69 & 96.3 & 2.50 & 2.50 & 52.7 & 1.8 \\
\hline s838 & 399 & 4.62 & 0.56 & 4.97 & 4.97 & 39.5 & 4.97 & 4.97 & 16.4 & 2.4 \\
\hline s1196 & 732 & 5.05 & 0.70 & 5.56 & 5.56 & 129.4 & 5.56 & 5.56 & 38.6 & 3.4 \\
\hline $\mathrm{s} 1238$ & 895 & 2.73 & 0.38 & 3.12 & 3.12 & 258.5 & 3.12 & 3.12 & 66.1 & 3.9 \\
\hline $\mathrm{s} 1423$ & 867 & 5.60 & 0.42 & 5.60 & 5.71 & 288.5 & 5.71 & 5.71 & 299.7 & 1.0 \\
\hline s1488 & 525 & 3.78 & 0.70 & 3.78 & 3.94 & 87.8 & 3.94 & 3.94 & 54.0 & 1.6 \\
\hline s1494 & 776 & 2.54 & 0.41 & 2.91 & 3.05 & 209.2 & 2.91 & 2.91 & 160.5 & 1.3 \\
\hline s5378 & 1403 & 3.78 & 0.70 & 4.28 & 4.28 & 850.8 & 4.28 & 4.28 & 300.0 & 2.8 \\
\hline s9234 & 1406 & 2.20 & 0.56 & 2.31 & 2.46 & 929.1 & 2.32 & 2.32 & 1123.3 & 0.8 \\
\hline s35932 & 11720 & 2.88 & 0.43 & 3.10 & 3.10 & 245944.3 & 3.10 & 3.10 & 23563.7 & 10.4 \\
\hline s38417 & 9750 & 6.19 & 0.70 & 6.19 & 6.19 & 16431.5 & 6.19 & 6.19 & 11675.8 & 1.4 \\
\hline average & & & & & 3.03 & & & 2.99 & & 2.1 \\
\hline
\end{tabular}

